\title{
Stretching Instability of Helical Springs
}

\author{
David A. Kessler and Yitzhak Rabin \\ Dept. of Physics, Bar-Ilan University, Ramat-Gan, Israel
}

(Dated: October 31, 2018)

\begin{abstract}
We show that when a gradually increasing tensile force is applied to the ends of a helical spring with sufficiently large ratios of radius to pitch and twist to bending rigidity, the end-to-end distance undergoes a sequence of discontinuous stretching transitions. Subsequent decrease of the force leads to step-like contraction and hysteresis is observed. For finite helices, the number of these transitions increases with the number of helical turns but only one stretching and one contraction instability survive in the limit of an infinite helix. We calculate the critical line that separates the region of parameters in which the deformation is continuous from that in which stretching instabilities occur, and propose experimental tests of our predictions.
\end{abstract}

PACS numbers: $46.32 .+\mathrm{x}, 46.25 . \mathrm{Cc}, 62.20 . \mathrm{Fe}$

The study of mechanical instability of thin elastic rods goes back to Euler and his contemporaries who considered the buckling of such rods under compression and torque 1, 2. The present paper presents a theoretical prediction of a hitherto unknown instability that arises when a helical spring is stretched by a tensile force applied to its ends. As we will show in the following, stretching instabilities occur only in helices whose radius is sufficiently larger than their pitch and whose rigidity with respect to twist exceeds that with respect to bending.

Consider a helical spring of contour length $L$, characterized by constant spontaneous curvature $\kappa_{0}$ and torsion $\tau_{0}$ or, alternatively, by its radius $r=\kappa_{0} /\left(\kappa_{0}^{2}+\tau_{0}^{2}\right)$ and pitch $p=2 \pi \tau_{0} /\left(\kappa_{0}^{2}+\tau_{0}^{2}\right)$. Under the action of a constant stretching force $F$ applied to its ends and directed along the z-axis (the ends are otherwise unconstrained and no torque is applied), the helix deforms into a curve whose shape is determined by minimizing the elastic energy [3, [4, 5, 6]

$$
E=\frac{1}{2} \sum_{i=1}^{3} a_{i} \int_{0}^{L} d s\left[\delta \omega_{i}(s)\right]^{2}-F R_{z},
$$

with respect to the deviations $\delta \omega_{i}(s)$ of the generalized curvatures and torsions $\omega_{i}(s)$ from their spontaneous values in the undeformed state ( $s$ is the contour parameter). In general, these parameters are related to the curvature $\kappa$, torsion $\tau$, and the angle $\alpha$ between one of the principal axes of the cross section $\left(\mathbf{t}_{1}\right)$ and the binormal: $\omega_{1}=\kappa \cos \alpha$, $\omega_{2}=\kappa \sin \alpha$ and $\omega_{3}=\tau+d \alpha / d s$. We study here the case that the only contribution to spontaneous twist comes from torsion $\left(\alpha_{0}=0\right)$ and therefore $\omega_{01}=\kappa_{0}, \omega_{02}=0$ and $\omega_{03}=\tau_{0}$. The coefficients $a_{1}$ and $a_{2}$ are bending rigidities associated with the principal axes of inertia of the (in general, noncircular) cross section and $a_{3}$ is the twist rigidity. In the following we treat $a_{i}$ as given material parameters of the spring. The shape of the deformed spring can be obtained by inserting the $\left\{\omega_{i}(s)\right\}$ that minimize the elastic energy into the generalized Frenet equations for the unit vectors $\mathbf{t}_{i}$ ( $\mathbf{t}_{3}$ is the tangent to the curve at point $s$ and $\mathbf{t}_{1}$ and $\mathbf{t}_{2}$ point along the principal axes of symmetry of the cross section):

$$
d \mathbf{t}_{i} / d s=-\sum_{j k} e_{i j k} \omega_{j} \mathbf{t}_{k},
$$

where $e_{i j k}$ is the antisymmetric unit tensor [7]. The space curve $\mathbf{r}(s)$ associated with the centerline of the deformed spring is then obtained by integrating the relation $d \mathbf{r} / d s=\mathbf{t}_{3}$. The system of equations is closed by substituting the expression for the projection of the end-to-end vector on the z-axis, $R_{z}=\int_{0}^{L} d s \mathbf{t}_{3}(s) \cdot \mathbf{z}$, into Eq. 1. Since the spring will always orient itself along the direction of the force, in the following we replace $R_{z}$ by the end-to end-distance $R$.

The force-extension curves are calculated numerically for a helix of 4 turns by finding a set of $\omega_{i}\left(s_{j}\right)$ (the continuous curve is replaced by a discrete set of points, $\left.\left\{s_{j}\right\}, j=1,2, \ldots\right)$ that minimizes the energy, Eq. 11. Two typical types of behavior are found. For sufficiently large ratio of pitch to radius $p / r=2 \pi \tau_{0} / \kappa_{0}$ and sufficiently small ratio of twist to bending rigidity $a_{3} / a_{b}$ (where $a_{b}^{-1}=\left(a_{1}^{-1}+a_{2}^{-1}\right) / 2$ ), the deformation is continuous and identical curves are obtained by stretching the undeformed helix and by starting from a fully stretched configuration and decreasing the tensile force to zero (Fig. 1).

In the opposite limit (sufficiently large $r / p$ and $a_{3} / a_{b}$ ratios), increasing $F$ results in a sequence of discontinuous upward jumps of the end-to-end distance (Fig. 2), whose number depends on the number of helical turns and on the increment of the force. Comparison of the conformations on both sides of a discontinuity shows that the jumps are associated with the elimination of helical turns (Fig. 2, insets). Discontinuous downward jumps of the end-to-end 


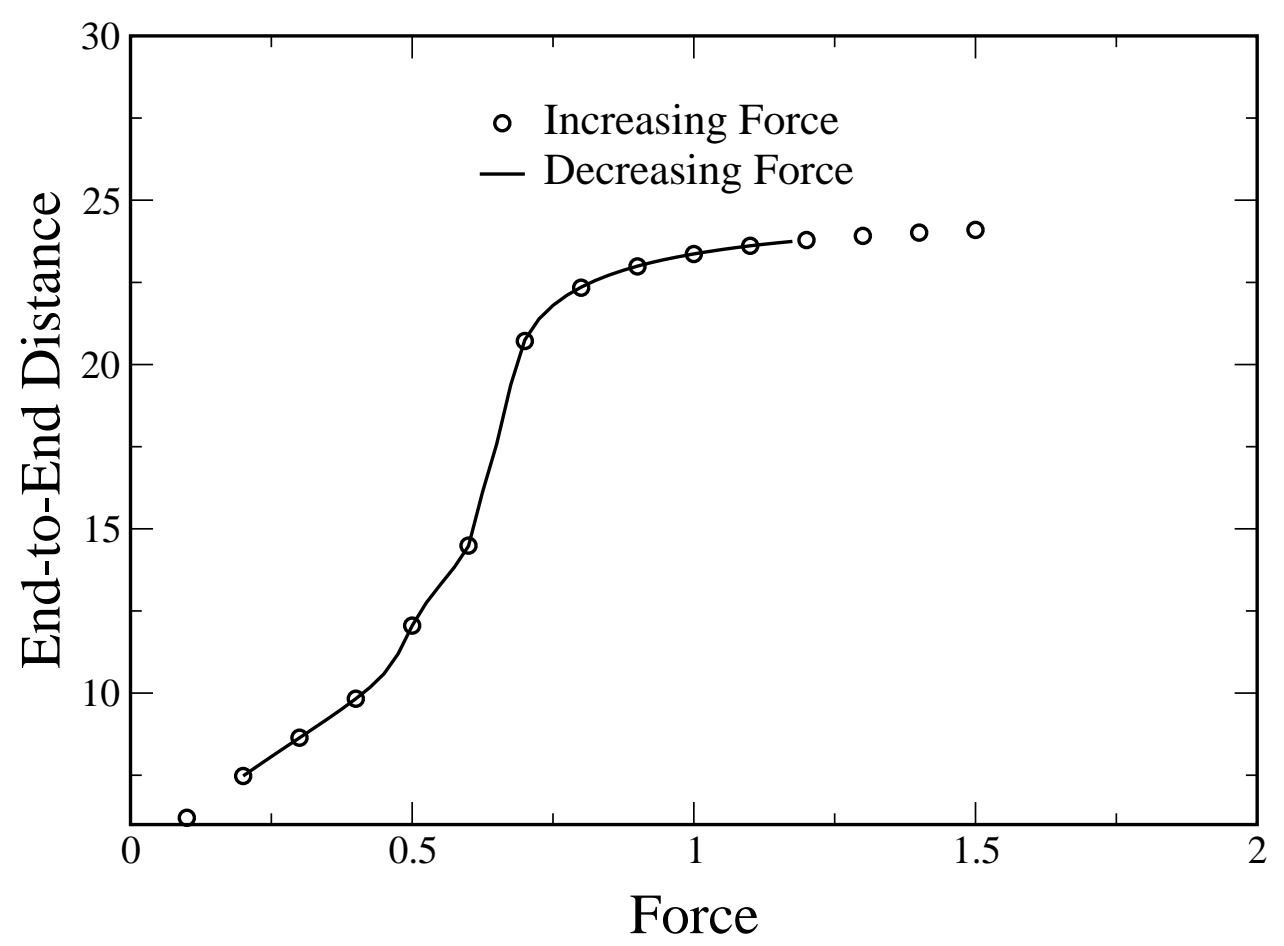

FIG. 1: Continuous deformation of a helix of 4 turns with $\kappa_{0}=1, \tau_{0}=0.2, a_{1}=1, a_{2}=5, a_{3}=3$. We show the end-to-end distance as a function of the force for both increasing (circles) and decreasing (solid line) force.

distance are also observed when one starts from a fully stretched configuration and gradually decreases the tensile force but the locations of these jumps are, in general, different from those of the upward ones. The observation of hysteresis loops of stretching and contraction suggests an analogy with first order phase transitions and implies that the elastic energy $E(R)$ has multiple local minima whose depth and location vary with the tensile force $F$. In the absence of thermal fluctuations, a transition to a new energy minimum (not necessarily the lowest energy one) takes place when the minimum corresponding to the original state disappears. While in systems undergoing phase transitions the choice of the final state is determined by the kinetics of the temperature quench, in our case it depends on the magnitude of the increment of the force.

The analogy with phase transitions suggests that there exists a critical surface in the parameter space that separates between regions in which the deformation is continuous from those in which a discontinuous stretching transition takes place. It is of particular interest to locate this critical surface in the limit of an infinitely long helix, for which end effects are negligible and it is expected that the only stable states are perturbations of the original undeformed helix and of the completely stretched spring (and therefore only one hysteresis loop that corresponds to a single stretching and a single contraction instabilities, is expected). Insight into this limit can be achieved via analytical approximations. In order to reduce the number of variables, we consider a ribbon with highly asymmetric cross section, $a_{2} / a_{1} \rightarrow \infty$, in which case the cross section of the ribbon is pinned to the Frenet frame of its centerline, $\alpha(s)=0$, and the deformed spring is completely characterized by $\kappa$ and $\tau$. Based on our numerical studies of short helices, we would like to stress that varying $a_{2}$ (while keeping all other parameters fixed) affects the detailed extension versus force curves but did not change the character of the transitions, and stretching instabilities accompanied by hysteresis were observed even in the symmetric case, $a_{1}=a_{2}$.

In the following we measure the energy in units of $a_{1} \kappa_{0}^{2} L$, and force in units of $a_{1} \kappa_{0}^{2}$. In order to obtain analytical results, we consider the limit of an infinitely long helix and assume that the spring maintains a helical shape and responds to deformation only by adjusting its curvature and torsion parameters. Thus, we look for constant $(s-$ independent) parameters $\kappa$ and $\tau$ that minimize the energy per unit length,

$$
E / L=\frac{1}{2}\left(\kappa / \kappa_{0}-1\right)^{2}+\frac{a_{3} / a_{1}}{2}\left(\tau / \kappa_{0}-\tau_{0} / \kappa_{0}\right)^{2}-\frac{F \tau / \kappa_{0}}{\sqrt{\left(\kappa / \kappa_{0}\right)^{2}+\left(\tau / \kappa_{0}\right)^{2}}} .
$$

Support for this variational approximation comes from numerical minimization of the energy, Eq. 1, which shows that while the curvature and torsion oscillate about their mean values, the amplitude of oscillation decreases with the 


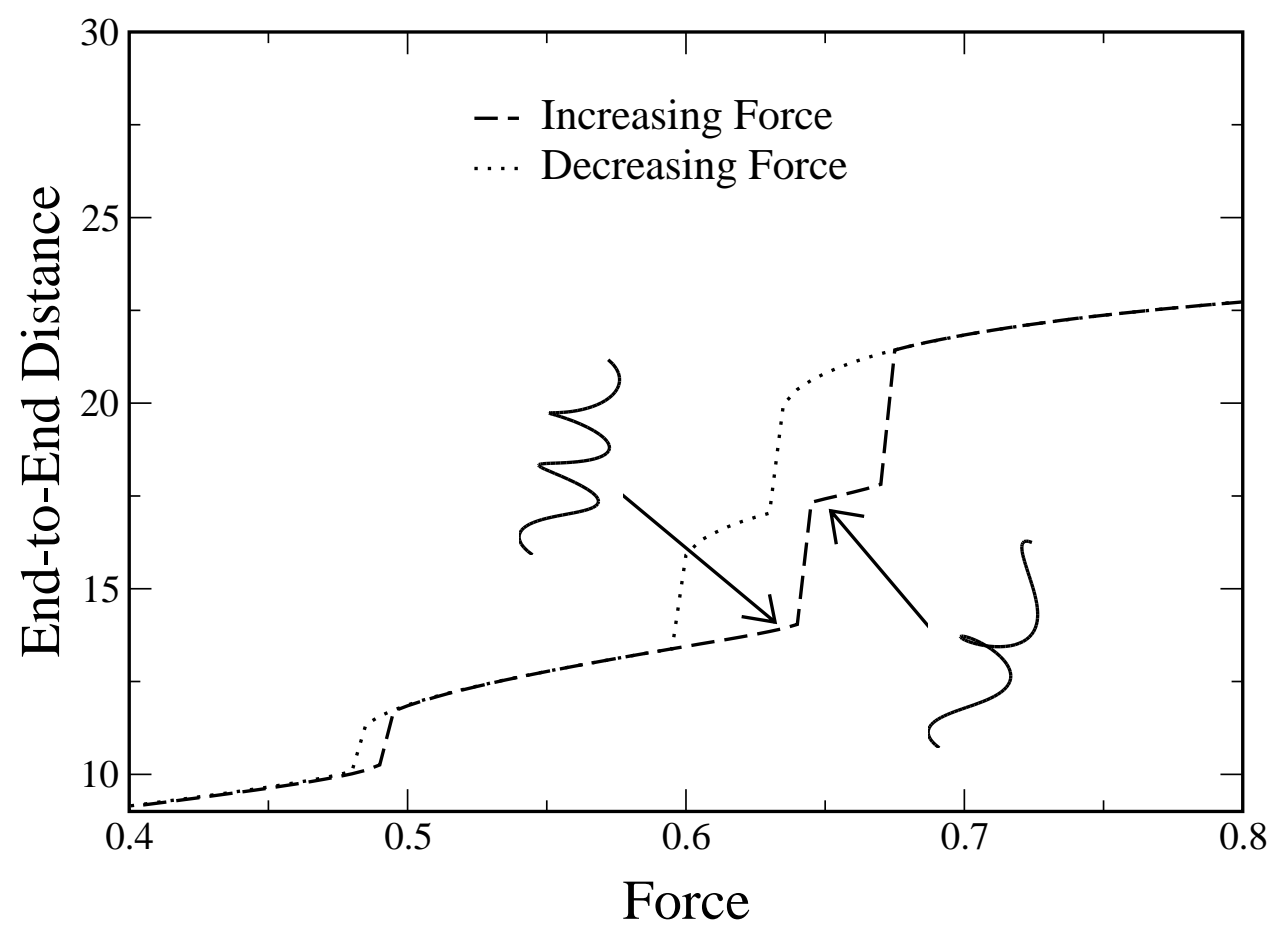

FIG. 2: Hysteresis loop for a helix of 4 turns with $\kappa_{0}=1, \tau_{0}=0.2, a_{1}=1, a_{2}=5, a_{3}=4$. We show the end-to-end distance as a function of the force for both increasing and decreasing force.

length of the spring. In Fig. 3 we compare the extension vs. force curve for a helix of 12 turns, obtained by exact numerical minimization of Eq. 1, with that obtained using the variational estimate. The fact that the two curves coincide up to the stability limits of the initial and the final states provides further support for the validity of our variational approach.

Analytical results can be obtained in several limiting cases. In the case of large twist rigidity, $a_{3} / a_{1} \rightarrow \infty$, the torsion is pinned to its spontaneous value $\left(\tau=\tau_{0}\right)$ and the curvature obeys the relation $a_{1}\left(\kappa / \kappa_{0}-1\right)+$ $F\left(\tau_{0} / \kappa_{0}\right)\left(\kappa / \kappa_{0}\right)\left[\left(\kappa / \kappa_{0}\right)^{2}+\left(\tau / \kappa_{0}\right)^{2}\right]^{-3 / 2}=0$. Graphical analysis of this equation shows that it admits either a single minimum or two minima separated by a maximum. For $\tau_{0}>\tau_{0, c}$ there is a single minimum of $\kappa$ as a function of $F$. For $\tau_{0}<\tau_{0, c}$, there is a window of $F$ between which there are 3 solutions and outside which there is only one solution. This window closes at the critical point: $\tau_{0, c} / \kappa_{0}=(2 / 3)^{5 / 3}=0.363, F_{c}=0.651$, and $\kappa_{c} / \kappa_{0}=0.444$. Another limit in which the critical point can be calculated analytically for arbitrary $a_{3} / a_{1}$ is $\tau_{0} / \kappa_{0} \ll 1$. In this case we find that two minima exist for $a_{3} / a_{1}>4 / 3$. The critical value of this ratio is therefore $4 / 3$ and at this point $F_{c}=4 / 3$, and $\kappa_{c} / \kappa_{0} \simeq 54^{1 / 5}\left(\tau_{0} / \kappa_{0}\right)^{2 / 5} / 2$. In between these two limits the critical points have to be found numerically, resulting in a critical line in the $a_{3} / a_{1}-\tau_{0} / \kappa_{0}$ plane (Fig. 4).

We have shown that there exists a range of spontaneous curvatures and torsions and bending and twist rigidities in which a helical spring does not deform continuously with the tensile force. Instead, as the force is increased, the spring undergoes a sequence of stretching instabilities. Hysteresis is predicted to take place when the force is decreased starting from full extension, as both the number of sudden contractions and their locations differ from that of the stretching instabilities at increasing force. We presented a variational calculation of the critical line that separates the region of parameters in which the deformation varies continuously with the force from that in which stretching instabilities take place. Even though the analysis was restricted to the limit of an infinitely long spring with a highly asymmetric cross section, our numerical results indicate that stretching instabilities will be observed in helical springs with both the radius to pitch ratio and the ratio of twist rigidity to bending rigidity larger than some numbers of order unity. While the former requirement can be realized by preparing a spring of a desired shape, the latter condition may require the use of materials with special elastic properties. Notice that for isotropic materials the rigidities can be expressed in terms of the shear and Young moduli $\mu$ and $E$ and the geometry of the cross section; for example, in the case of rectangular cross section with sides $c_{1}$ and $c_{2}$ one has $\left[a_{1}=E c_{1} c_{2}^{3} / 12, a_{2}=E c_{1}^{3} c_{2} / 12\right.$, and $a_{3} \simeq \mu c_{1} c_{2}^{3} / 3$ (the last expression holds in the limit $c_{1} \gg c_{2}$ ). For incompressible materials this yields $a_{3} / a_{1} \simeq 4 / 3$ which coincides 


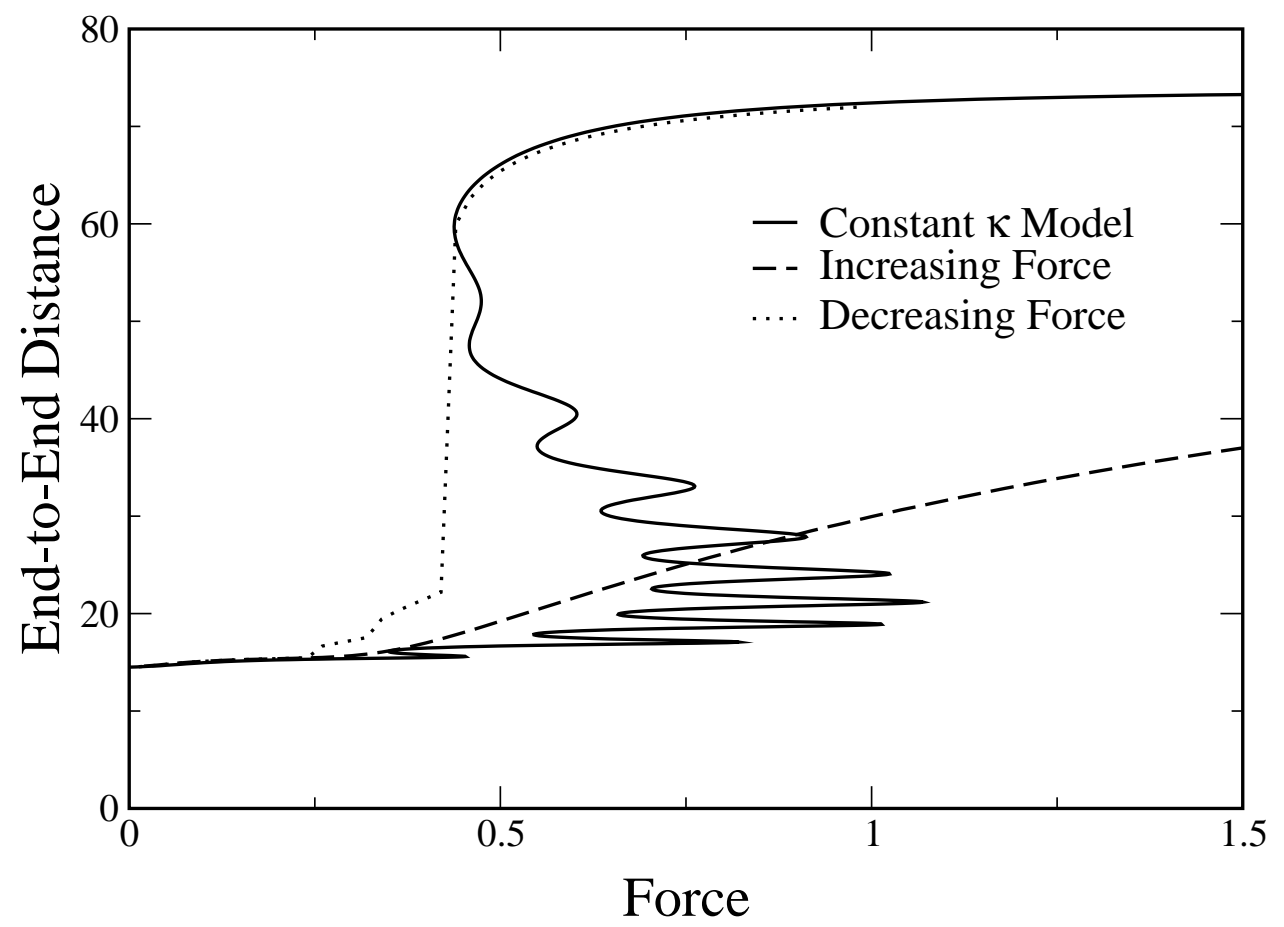

FIG. 3: Hysteresis loop for a helix of 12 turns, with $\kappa_{0}=1, \tau_{0}=0.2, a_{1}=1, a_{2}=a_{3}=\infty$. We show the end-to-end distance as a function of the force for both increasing (dashed line) and decreasing (dotted line) force. Also shown is the result of our simple model with constant $\kappa$ (solid line).

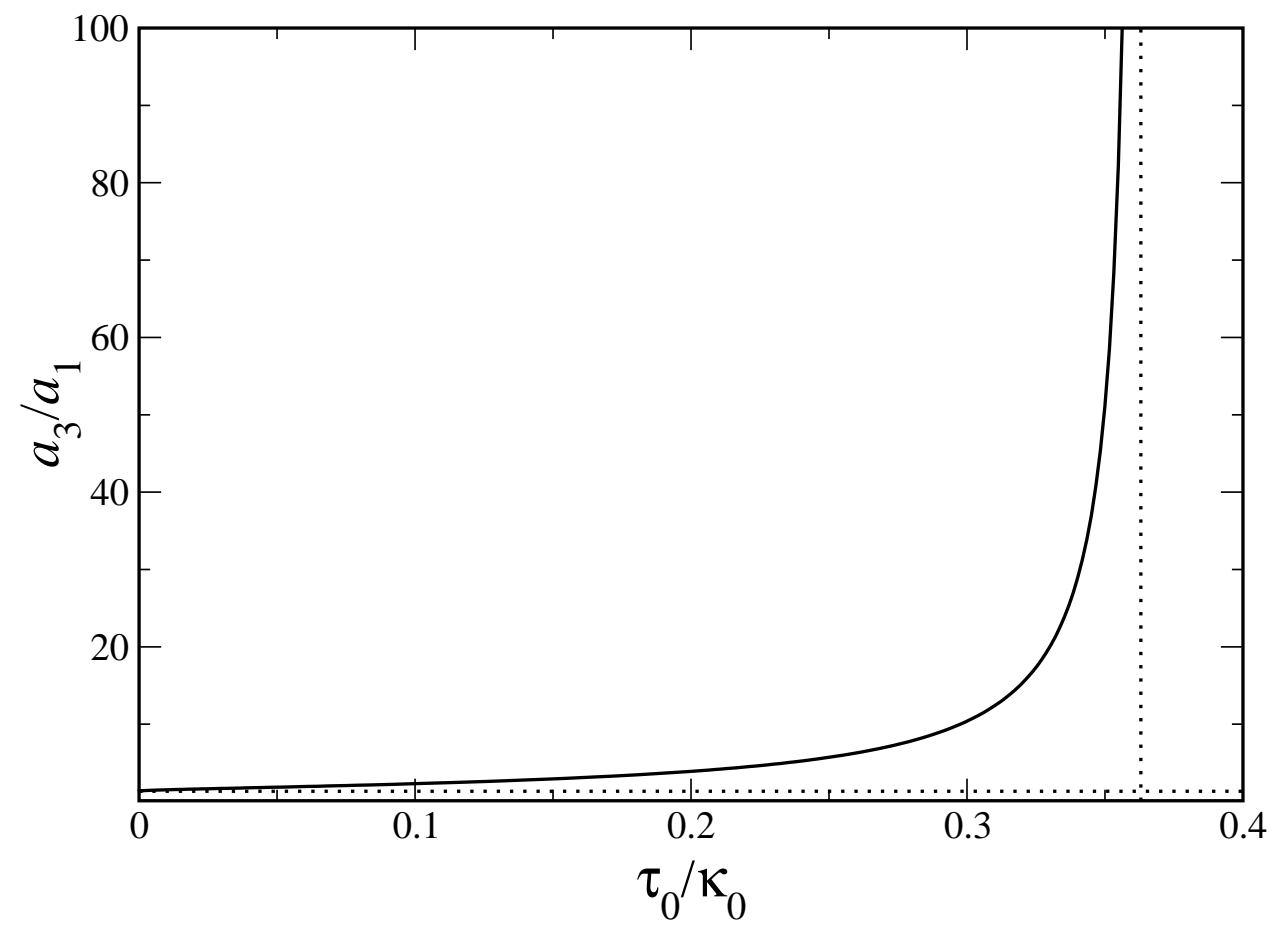

FIG. 4: Critical line in the $a_{3} / a_{1}-\tau_{0} / \kappa_{0}$ plane for an infinitely long helix with $a_{2} / a_{1} \rightarrow \infty$. The vertical and horizontal dotted lines correspond to the two limits discussed in the text, $\tau_{0, c} / \kappa_{0}=0.363$ and $a_{3} / a_{1}=1.333$, respectively. 
with the critical value of the ratio. The ratio $a_{3} / a_{1}$ can be increased beyond this critical value by reducing the Poisson ratio or by using anisotropic materials with high resistance to twist.

We would like to comment on the connection between the stretching instabilities of helical springs and those familiar from other branches of physics. The occurrence of multiple energy minima and hysteresis bring to mind first order phase transitions but since thermal fluctuations and entropy play no role in our work, the analogy is somewhat misleading. The predicted transitions appear to be more closely related to hydrodynamic instabilities, even though the latter arise due to nonlinear effects, while the former can be described within the framework of the linear theory of elasticity [9]. Further reflection shows that the elastic theory of thin rods is truly linear only in the "Lagrangian" frame of the deformed object (i.e., in its intrinsic coordinate description). In the presence of external force one must transform into the laboratory frame (e.g., Euler angles) and the energy is no longer a quadratic form in the deviations from the undeformed state (recall that hydrodynamics is also a linear theory in the Lagrangian frame!).

Finally, we would like to stress that since the present analysis is purely mechanical, the results are directly applicable only to macroscopic objects. The relevance of the present work to the deformation of nano-objects such as biopolymers (DNA, proteins), protein filaments (actin, microtubules, etc.), and carbon nanotubes depends on the question of whether these objects possess spontaneous curvature and torsion. Interestingly, discontinuous stretching transitions were reported in single molecule extension studies of torsionally constrained DNA at high degree of supercoiling (unlike our model, supercoiling was not spontaneous but was achieved by the application of torque) 10]. The study of stretching instabilities of such objects requires consideration of thermal fluctuations and extension of the present analysis using the methods of references [7] and [6], which we hope to report on soon.

DAK and YR acknowledge the support of the Israel Science Foundation.

[1] A.E.H. Love, A Treatise on the Mathematical Theory of Elasticity (Dover, New York, 1944).

[2] S.P. Timoshenko and J.M. Gere, Theory of Elastic Stability (McGraw-Hill, New York, 1963).

[3] Lord Kelvin and P.G. Tait, Treatise on Natural Philosophy (University Press, Cambridge, 1883).

[4] P. Nelson, Phys. Rev. Lett. 80, 5810 (1998).

[5] R.E. Goldstein, A. Goriely, G. Huber and C.W. Wolgemuth, Phys. Rev. Lett. 84, 1631 (2000).

[6] S. Panyukov and Y. Rabin, Europhys. Lett. 57, 512 (2002).

[7] S. Panyukov and Y. Rabin, Phys. Rev. Lett. 85, 2404 (2000); Phys. Rev. E 62, 7135 (2000).

[8] L.D. Landau and E.M. Lifshitz, Theory of Elasticity (Pergamon Press, Oxford, 1986).

[9] Recall that for thin rods linear elasticity applies even for large global deformations af a long rod, and that the only limitation is that the characteristic length scales associated with the deformation $\left(1 /\left|\omega_{i}\right|\right)$ are much larger than the diameter of the spring.

[10] T.R. Strick, J.-F. Allemand, D. Bensimon, A. Bensimon and V. Croquette, Science 271, 1835 (1996). 